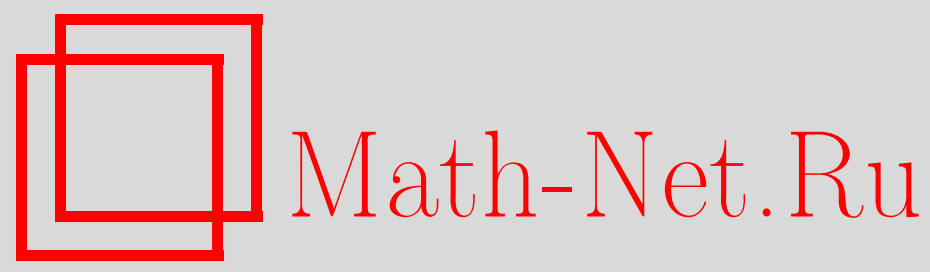

В. В. Арестов, Наилучшее приближение одного класса функций многих переменных другим и родственные экстремальные задачи, Матем. заметки, 1998, том 64, выпуск $3,323-340$

DOI: https://doi.org/10.4213/mzm1403

Использование Общероссийского математического портала Math-Net.Ru подразумевает, что вы прочитали и согласны с пользовательским соглашением http://www . mathnet.ru/rus/agreement

Параметры загрузки:

IP : 54.84 .234 .179

26 апреля 2023 г., 10:10:05

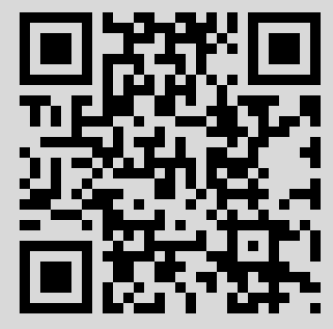




\title{
НАИЛУЧШЕЕ ПРИБЛИЖЕНИЕ ОДНОГО КЛАССА ФУНКЦИЙ МНОГИХ ПЕРЕМЕННЫХ ДРУГИМ И РОДСТВЕННЫЕ ЭКСТРЕМАЛЬНЫЕ ЗАДАЧИ
}

В. В. Арестов

\begin{abstract}
В работе исследована взаимосвязь нескольких экстремальных задач для неограниченных линейных операторов типа свертки в пространствах $L_{\gamma}=L_{\gamma}\left(\mathbb{R}^{m}\right), m \geqslant 1$, $1 \leqslant \gamma \leqslant \infty$. Для задачи о вычислении модуля непрерывности оператора свертки $A$ на классе функций $Q$, определенном вторым подобным оператором, и задачи Стечкина о наилучшем приближении оператора $A$ на классе $Q$ линейньми ограниченными операторами построены двойственные задачи в сопряженных пространствах, являющиеся соответственно задачами о наилучшем и наилучшем линейном приближении одного класса функций другим.
\end{abstract}

Библиография: 28 названий.

В данной работе исследуется взаимосвязь нескольких экстремальных задач для неограниченных линейных операторов типа свертки в пространствах $L_{\gamma}=L_{\gamma}\left(\mathbb{R}^{m}\right), m \geqslant 1$, $1 \leqslant \gamma \leqslant \infty$. Для задачи о вычислении модуля непрерьвности оператора свертки $A$ на классе функций $Q$, определенном вторым подобным оператором, и задачи Стечкина [1] о наилучшем приближении оператора $A$ на классе $Q$ линейными ограниченными операторами построены двойственные задачи в сопряженных пространствах, являюшиеся соответственно задачами о наилучшем и наилучшем линейном приближении одного класса функций другим. Для операторов дифференцирования на классах функций одного переменного в пространствах $L_{\gamma}(I)$ на оси $I=(-\infty, \infty)$ и полуоси $I=[0, \infty)$ подобные результаты были получены ранее автором [2], а для периодических функций-Б. Е. Клоцем [3]. В настоящее время рассматриваемым здесь задачам посвящено большое число работ; обзор результатов по этой проблематике имеется в [4], [5].

1. Введение. Постановка задач. Пусть $L_{\gamma}=L_{\gamma}\left(\mathbb{R}^{m}\right), m \geqslant 1$, при $1 \leqslant \gamma<\infty$ есть пространство вешественных измеримых функций $x$ на $\mathbb{R}^{m} \mathrm{c}$ (конечной) нормой

$$
\|x\|_{\gamma}=\left(\int_{\mathbb{R}^{m}}|x(t)|^{\gamma} d t\right)^{1 / \gamma},
$$

а при $\gamma=\infty$ (если не оговорено что-то иное) - пространство $C_{0}=C_{0}\left(\mathbb{R}^{m}\right)$ непрерывных на $\mathbb{R}^{m}$ функций, имеющих нулевой предел на бесконечности, с равномерной нормой $\|x\|_{\infty}=\|x\|_{C_{0}}=\max \left\{|x(t)|: t \in \mathbb{R}^{m}\right\}$.

Работа выполнена при финансовой поддержке фонда INTAS, грант № 94-4070. 
Пусть, далее, $\mathscr{S}=\mathscr{S}\left(\mathbb{R}^{m}\right)$ есть пространство быстро убывающих бесконечно дифференцируемых функций на $\mathbb{R}^{m}$ (пространство основных или пробных функций), а $\mathscr{S}^{\prime}=$ $\mathscr{S}^{\prime}\left(\mathbb{R}^{m}\right)$ - соответствующее пространство обобщенных функций (см., например, [6]-[8]). Значение функционала (обобщенной функции) $\theta \in \mathscr{S}^{\prime}$ на элементе $x \in \mathscr{S}$ будем обозначать через $\langle\theta, x\rangle$. Сверткой $\theta * x$ элементов $\theta \in \mathscr{S}^{\prime}$ и $x \in \mathscr{S}$ назьвают функцию $y(\eta)=\left\langle\theta, \sigma_{\eta} x\right\rangle$, где операция $\sigma_{\eta}, \eta \in \mathbb{R}^{m}$, определена формулой $\left(\sigma_{\eta} x\right)(t)=x(\eta-t)$. Для $\theta \in \mathscr{S}^{\prime}$ обозначим через $\check{\theta}$ функционал, который действует по формуле $\langle\check{\theta}, x\rangle=\left\langle\theta, \sigma_{0} x\right\rangle=$ $\langle\theta, \check{x}\rangle, \check{x}(t)=x(-t), x \in \mathscr{S}$; таким образом, это есть операция замены знака аргумента обобщенной функции.

Ниже в работе обсуждается несколько экстремальных задач для линейных неограниченных операторов типа свертки (с некоторой обобщенной функцией), построенных по следующей единой схеме. С помощью элемента $\theta \in \mathscr{S}^{\prime}$ определим на $\mathscr{S}$ оператор $\Theta$ и формально сопряженный оператор $\Theta *$ формулами

$$
\Theta x=\check{\theta} * x, \quad \Theta^{*} x=\theta * x, \quad x \in \mathscr{S} .
$$

Всегда будет предполагаться выполненным условие, что $\theta * x \in \mathscr{S}$ для любой функции $x \in \mathscr{S}$, т.е. $\Theta \mathscr{S} \subset \mathscr{S}$ (или, то же самое, $\Theta^{*} \mathscr{S} \subset \mathscr{S}$ ); это будет иметь место, например, в том случае, если $\theta$ имеет компактный носитель. Распространим оператор $\Theta$ на более широкое множество по схеме Соболева. Обозначим через $\Sigma=\Sigma\left(\mathbb{R}^{m}\right)$ множество измеримых, локально суммируемых функций на $\mathbb{R}^{m}$, для каждой из которых найдется показатель $\mu=\mu(x) \leqslant 0$ со свойством

$$
\int|x(t)|\left(1+|t|^{2}\right)^{\mu} d t<\infty
$$

здесь и ниже в интегралах по $\mathbb{R}^{m}$ множество интегрирования не указывается. Ясно, что $\Sigma \subset \mathscr{S}^{\prime}$. Относительно пары функций $x, y \in \Sigma$ будем считать, что $x$ принадлежит области определения $D(\Theta)$ оператора $\Theta$ и $y=\Theta x$, если для любой функции $\phi \in \mathscr{S}$ имеет место равенство

$$
\int x \Theta^{*} \phi d t=\int y \phi d t .
$$

Аналогичным образом можно осуществить продолжение оператора $\Theta^{*}$. Нетрудно понять, что функция $x$ принадлежит области определения $D\left(\Theta^{*}\right)$ оператора $\Theta^{*}$ в том и только том случае, если $\check{x} \in D(\Theta)$ и при этом $\left(\Theta^{*} x\right)(\eta)=(\Theta \check{x})(-\eta), \eta \in \mathbb{R}^{m}$. Операторы $\Theta$ и $\Theta^{*}$ инвариантны относительно сдвигов соответственно на $D(\Theta)$ и $D\left(\Theta^{*}\right)$, т.е. обладают свойствами, что $\tau_{\eta} \Theta=\Theta \tau_{\eta}$ и $\tau_{\eta} \Theta^{*}=\Theta^{*} \tau_{\eta}$ при любом $\eta \in \mathbb{R}^{m}$, где $\tau_{\eta}-$ оператор сдвига, определенньй (на множестве $\Sigma$ ) соотношением $\left(\tau_{\eta} x\right)(t)=x(t-\eta)$.

Пусть $A$ и $A^{*}$ есть пара (формально сопряженных) операторов с областями определения $D(A), D\left(A^{*}\right)$, построенных по только что изложенной схеме с помощью элемента $a \in \mathscr{S}^{\prime}$. Допустим, что $B$ и $B^{*}$ есть еще одна пара операторов с областями определения $D(B), D\left(B^{*}\right)$, определенных тем же путем с помощью еще одного элемента $b \in \mathscr{S}^{\prime}$. Предположим, что $p, q, r, s$ - параметры, удовлетворяющие условиям $1 \leqslant p, q, r, s \leqslant \infty$. Введем пространства

$$
\begin{gathered}
W_{p}(B)=\left\{x \in D(B): B x \in L_{p}\right\} \\
W_{r, p}(B)=L_{r} \cap W_{p}(B)=\left\{x \in L_{r} \cap D(B): B x \in L_{p}\right\} .
\end{gathered}
$$


На множестве $W_{r, p}(B)$ выделим класс

$$
Q=Q_{r, p}(B)=\left\{x \in W_{r, p}(B):\|B x\|_{p} \leqslant 1\right\} .
$$

Обозначим через $\mathscr{B}_{r}^{s}$ множество линейных ограниченных операторов из $L_{r}$ в $L_{s}$, и пусть $\mathscr{B}_{r}^{s}(N)$ есть множество операторов $T \in \mathscr{B}_{r}^{s}$, норма которых ограничена числом $N \geqslant 0$ : $\|T\|=\|T\|_{L_{r} \rightarrow L_{s}} \leqslant N$.

Одной из обсуждаемых в данной работе задач является задача С. Б. Стечкина о наилучшем приближении оператора $A$ на классе $Q$ в метрике пространства $L_{q}$ множеством линейных ограниченных операторов $T \in \mathscr{B}_{r}^{s}(N)$, т.е. задача об исследовании величины

$$
\mathscr{E}(N)=\mathscr{E}(A, B ; r, s ; p, q ; N)=\inf \left\{U(T): T \in \mathscr{B}_{r}^{s}(N)\right\}
$$

где

$$
U(T)=\sup \left\{\|A x-T x\|_{q}: x \in Q_{r, p}(B)\right\} ;
$$

при этом если $W_{r, p}(B) \not \subset D(A)$, то считаем, что $U(T)=\infty$ для любого оператора $T \in \mathscr{B}_{r}^{s}(N)$. В классическом варианте этой задачи [1] параметры $s$ и $q$ совпадают: $s=q$. В этом случае величину (2) будем обозначать через $E(N)$; таким образом, $E(N)=\mathscr{E}(A, B ; r, q ; p, q ; N)$. Задача (2) довольно подробно исследована, в частности, выяснена ее взаимосвязь с другими экстремальньми задачами, дано ее решение для конкретных операторов в классических функциональных пространствах (см. библиографию в [5]); ниже будут использоваться результаты работ автора [9]-[13], относящиеся к этой задаче. Функцию

$$
\omega(\delta)=\sup \left\{\|A x\|_{q}: x \in Q_{r, p}(B),\|x\|_{r} \leqslant \delta\right\}
$$

переменного $\delta \in[0, \infty)$ назьвают модулем непрерывности оператора $A$ на классе $Q=$ $Q_{r, p}(B)$. Положим

$$
\begin{gathered}
R(N)=\sup \{\omega(\delta)-N \delta: \delta \geqslant 0\}=\sup \left\{\|A x\|_{q}-N\|x\|_{r}: x \in Q_{r, p}(B)\right\}, \\
\lambda(\delta)=\inf \{E(N)+N \delta: N \geqslant 0\} .
\end{gathered}
$$

С. Б. Стечкин [1] заметил, что имеют место неравенства

$$
\begin{gathered}
E(N) \geqslant R(N), \quad N \geqslant 0, \\
\omega(\delta) \leqslant \lambda(\delta), \quad \delta \geqslant 0 ;
\end{gathered}
$$

если при этом существует элемент $x \in Q$ и линейный ограниченньй оператор $T \in \mathscr{B}_{r}^{q}$ со свойствами

$$
\|A x\|=U(T)+\|T\| \cdot\|x\|,
$$

то имеют место равенства

$$
\omega(\|x\|)=\|A x\|, \quad E(\|T\|)=U(T)=\|A x\|-\|T\| \cdot\|x\|,
$$

и, следовательно, оператор $T$ является экстремальным в задаче $E(N)$ при $N=\|T\|=$ $\|T\|_{L_{r} \rightarrow L_{q}}$, а элемент $x$ - в задаче (3) при $\delta=\|x\|$. Это утверждение дает простую, но 
часто используемую и эффективную оценку снизу величины наилучшего приближения оператора через его модуль непрерьвности.

В настояшее время особенно полно изучены задачи (2) и (3) для операторов дифференцирования $D^{k} x=x^{(k)}$ порядка $k$ на классе

$$
Q=Q_{r, p}^{n}=\left\{x \in L_{r}: x^{(n)} \in L_{p},\left\|x^{(n)}\right\|_{p} \leqslant 1\right\}
$$

$n$ раз дифференцируемых функций $(0 \leqslant k<n)$ в пространствах $L_{\gamma}=L_{\gamma}(I), 1 \leqslant \gamma \leqslant \infty$, функций одного переменного на числовой оси $I=(-\infty, \infty)$ и полуоси $I=[0, \infty)($ см. библиографию в [4], [5]). В этом случае

$$
\begin{gathered}
E(N)=E_{n, k}(N)=\inf \left\{U(T):\|T\|_{L_{r} \rightarrow L_{q}} \leqslant N\right\} \\
U(T)=\sup \left\{\left\|x^{(k)}-T x\right\|_{q}: x \in Q_{r, p}^{n}\right\} .
\end{gathered}
$$

Известно, что если вьполнено условие $k+1 / r-1 / q>0$, то имеет место равенство

$$
E_{n, k}(N)=N^{-\gamma} E_{n, k}(1), \quad \gamma=\frac{n-k+1 / q-1 / p}{k+1 / r-1 / q} .
$$

В данной ситуации функция (3) имеет вид

$$
\omega(\delta)=\sup \left\{\left\|x^{(k)}\right\|_{q}:\|x\|_{r} \leqslant \delta,\left\|x^{(n)}\right\|_{p} \leqslant 1\right\}
$$

при выполнении условия $n-1 / p+1 / r>0$ для нее справедлива формула

$$
\omega(\delta)=K \delta^{\alpha}, \quad K=\omega(1), \quad \alpha=\frac{n-k-1 / p+1 / q}{n-1 / p+1 / r} .
$$

Величина $K=\omega(1)$ есть наименьшая (наилучшая) константа в известном неравенстве между нормами производных функций (неравенстве Колмогорова)

$$
\left\|x^{(k)}\right\|_{q} \leqslant K\|x\|_{r}^{\alpha}\left\|x^{(n)}\right\|_{p}^{\beta}, \quad \alpha=\frac{n-k-1 / p+1 / q}{n-1 / p+1 / r}, \quad \beta=1-\alpha .
$$

Если $k+1 / r-1 / q>0$, то неравенство (4) Стечкина превращается в следующее соотношение между величиной $E_{n, k}(N)$ и наилучшей константой $K$ в неравенстве $(6)$ :

$$
E_{n, k}(N) \geqslant \beta \alpha^{\alpha / \beta} K^{1 / \beta} N^{-\alpha / \beta}, \quad N>0 .
$$

Существует связь задач (3) и (2) с задачей приближения одного класса функций другим и с соответствующей задачей линейного приближения класса классом. Пусть $p^{\prime}, q^{\prime}, r^{\prime}$ - параметры, удовлетворяющие условиям $1 \leqslant p^{\prime}, q^{\prime}, r^{\prime} \leqslant \infty$ и не связанные пока никак с параметрами $p, q, r$. С помощю элемента $h \in \mathscr{S}^{\prime}$ определим по изложенной выше схеме еще один оператор $H$. В пространствах

$$
W_{r^{\prime}}(B)=\left\{\varphi \in D(B): B \varphi \in L_{r^{\prime}}\right\}, \quad W_{q^{\prime}}(H)=\left\{\psi \in D(H): H \psi \in L_{q^{\prime}}\right\}
$$


выделим классы

$$
\begin{gathered}
Q_{r^{\prime}}(B)=\left\{\varphi \in W_{r^{\prime}}(B):\|B \varphi\|_{r^{\prime}} \leqslant 1\right\}, \\
Q_{2}=N Q_{r^{\prime}}(B)=\left\{\varphi \in W_{r^{\prime}}(B):\|B \varphi\|_{r^{\prime}} \leqslant N\right\}, \\
Q_{1}=Q_{q^{\prime}}(H)=\left\{\psi \in W_{q^{\prime}}(H):\|H \psi\|_{q^{\prime}} \leqslant 1\right\} .
\end{gathered}
$$

Положим

$$
F(N)=F\left(Q_{1}, Q_{2}\right)_{p^{\prime}}=F\left(Q_{q^{\prime}}(H), N Q_{r^{\prime}}(B)\right)_{p^{\prime}}=\sup \left\{F\left(\psi, N Q_{r^{\prime}}(B)\right)_{p^{\prime}}: \psi \in Q_{q^{\prime}}(H)\right\}
$$

где

$$
F\left(\psi, N Q_{r^{\prime}}(B)\right)_{p^{\prime}}=\inf \left\{\|\psi-\varphi\|_{\widetilde{L}_{p^{\prime}}}: \varphi \in N Q_{r^{\prime}}(B)\right\} .
$$

Величину (7) называют величиной наилучшего приближения (или наилучиим приближсением в п пространстве $L_{p^{\prime}}$ класса $Q_{1}=Q_{q^{\prime}}(H)$ классом $Q_{2}=N Q_{r^{\prime}}(B)$. Hauлучшим линейным приближсением в $L_{p^{\prime}}$ класса $Q_{1}$ классом $Q_{2}$ называют величину

$$
G(N)=G\left(Q_{1}, Q_{2}\right)_{p^{\prime}}=G\left(Q_{q^{\prime}}(H), N Q_{r^{\prime}}(B)\right)_{p^{\prime}}=\inf \left\{G\left(Q_{q^{\prime}}(H) ; P\right)_{p^{\prime}}: P \in \mathscr{P}(N)\right\},
$$

где

$$
G\left(Q_{q^{\prime}}(H) ; P\right)_{p^{\prime}}=\sup \left\{\|\psi-P \psi\|_{p^{\prime}}: \psi \in Q_{q^{\prime}}(H)\right\},
$$

и нижняя грань в (8) берется по множеству $\mathscr{P}(N)$ всех линейных отображений $P$ класса $Q_{1}$ в класс $Q_{2}$.

Связь задач $(7),(8)$ с задачами (3), (2) была замечена впервые на семинаре С. Б. Стечкина в Свердловском отделении Математического института им В. А. Стеклова (ныне Институт математики и механики УрО РАН) в 1965-67 годах в связи с изучением задачи Стечкина (5) для операторов дифференцирования и неравенств Колмогорова (6) для классов функций одного переменного на оси и полуоси. Основное наблюдение состояло в том, что величину $G(N)$ наилучшего линейного приближения класса классом, а значит, и величину $F(N)$ наилучшего приближения класса классом можно оценить сверху через величину $E(N)$; полученные в этой проблематике результаты содержатся в работах [14]-[18]. Изложим суть этого подхода в применении к рассматриваемьм нами задачам. Допустим, что вьполнены предположения.

1) Параметры задач связаны условиями $p^{\prime}=q, q^{\prime}=p, r^{\prime}=q$.

2) Оператор $B$ является композицией оператора $A$ и оператора $H$, т.е. $B=H \circ A$; точнее, имеют место соотношения

$$
D(B) \subset D(A), \quad A D(B) \subset D(H), \quad H A x=B x, \quad x \in D(B) .
$$

Это предположение в дальнейшем мы будем называть условием факторизации $(\Phi)$.

3) Множество $A W_{p}(B)$ является достаточно богатым подмножеством пространства $W_{p}(H)$; для простоты рассуждений ограничимся случаем

$$
A W_{p}(B)=W_{p}(H) .
$$

4) В задаче (2) можно ограничиться множеством $\mathscr{B}^{\prime}$ операторов $T \in \mathscr{B}_{p, q}$, которые определены, линейные и перестановочны с оператором $B$ на множестве $W_{p}(B)$ (например, в задаче (2) существует экстремальньй оператор $T$ с такими свойствами). Более 
того, для любого такого оператора $T \in \mathscr{B}^{\prime}$ величина $\bar{U}(T)=\sup \left\{\|A x-T x\|_{q}: x \in Q_{p}(B)\right\}$ совпадает с величиной $U(T)=\sup \left\{\|A x-T x\|_{q}: x \in Q=Q_{p, p}(B)\right\}$.

При сделанных предположениях имеет место неравенство

$$
G(N)=G\left(Q_{p}(H), N Q_{q}(B)\right)_{q} \leqslant E(N)=\mathscr{E}(A, B ; p, q ; p, q ; N)
$$

а значит, и неравенство $F(N) \leqslant E(N)$. Действительно, оператору $T \in \mathscr{B}^{\prime}$ сопоставим оператор $P \in \mathscr{P}(N), N=\|T\|_{L_{p} \rightarrow L_{q}}$, по следующему правилу. В силу свойства $(9)$ для любой функции $\psi \in W_{p}(H)$ существует функция $x \in W_{p}(B)$ такая, что $A x=\psi$. Определим оператор $P$, положив $P \psi=T x, \psi \in W_{p}(H)$. Имеем $B P \psi=B T x=T B x=T H \psi$. Поэтому $\|B P \psi\|_{q} \leqslant\|T\|_{L_{p} \rightarrow L_{q}}\|H \psi\|_{p}$ и, значит, $P \in \mathscr{P}(N), N=\|T\|_{L_{p} \rightarrow L_{q}}$. Далее, находим

$$
\|\psi-P \psi\|_{q}=\|A x-T x\|_{q} \leqslant \bar{U}(T)\|B x\|_{p}=\bar{U}(T)\|H \psi\|_{p},
$$

а отсюда в силу четвертого предположения следует неравенство (10).

На самом деле естественной является иная связь введенных задач. А именно (при определенных условиях на операторы и правильньх соотношениях между параметрами), задача приближения класса классом является двойственной для задачи (3), а линейная задача приближения класса классом является двойственной для задачи (2). Впервые этот факт был замечен автором для операторов дифференцирования $D^{k}$ на оси и полуоси [2]; для периодических функций аналогичньй результат получил Б. Е. Клоц [3].

Задачи приближения класса классом будут рассматриваться в сопряженных пространствах по отношению к задачам (3) и (2). С этого момента мы будем считать, что параметры задач связаны условиями (Г) сопряженности по Гёльдеру, а точнее,

$$
\frac{1}{p}+\frac{1}{p^{\prime}}=1, \frac{1}{q}+\frac{1}{q^{\prime}}=1, \frac{1}{r}+\frac{1}{r^{\prime}}=1 .
$$

Возникает необходимость уточнить (изменить) определение некоторых классов функций. В случае $\gamma=\infty$ наряду с пространством $L_{\infty}=C_{0}$ будут рассматриваться еще два: пространство $\widetilde{L}_{\infty}=\widetilde{L}_{\infty}\left(\mathbb{R}^{m}\right)$ измеримых существенно ограниченных функций на $\mathbb{R}^{m}$ и пространство $C=C\left(\mathbb{R}^{m}\right)$ непрерьвных ограниченных функций с равномерной нормой. Иногда для пространств $L_{\gamma}=L_{\gamma}\left(\mathbb{R}^{m}\right)$ при $1 \leqslant \gamma<\infty$ нам будет удобно использовать также обозначения $\widetilde{L}_{\gamma}=\widetilde{L}_{\gamma}\left(\mathbb{R}^{m}\right)$. Пусть $M=M\left(\mathbb{R}^{m}\right)$ есть пространство (знакопеременных) $\sigma$-аддитивных конечных мер Лебега на $\mathbb{R}^{m}$; для обозначения вариации меры $\Xi \in M$ будет использоваться стандартное обозначение $\bigvee \Xi$. Пространство $L_{\rho}^{*}$ ограниченных линейных функционалов на пространстве $L_{\rho}$ в случае $1 \leqslant \rho<\infty$, как известно, совпадает с пространством $L_{\rho^{\prime}}, 1 / \rho+1 / \rho^{\prime}=1$, если $1<\rho<\infty$, и с пространством $\widetilde{L}_{\infty}$, если $\rho=1$ (т.е. с пространством $\widetilde{L}_{\rho^{\prime}}$ при всех $1 \leqslant \rho<\infty$ ), а точнее, функционал $\Upsilon \in L_{\rho}^{*}$ имеет вид

$$
\Upsilon x=\int x(t) \xi(t) d t,
$$

где $\xi \in \widetilde{L}_{\rho^{\prime}}$ и $\|\Upsilon\|_{L_{\rho}^{*}}=\|\xi\|_{\rho^{\prime}}$ В В случае $\rho=\infty$ пространство $L_{\infty}^{*}=C_{0}^{*}$ есть пространство мер $M=M\left(\mathbb{R}^{m}\right)$, и каждый функционал $\Upsilon \in C_{0}^{*}$ представляется в виде

$$
\Upsilon x=\int x(t) d \Xi(t)
$$


где $\Xi \in M$, при этом $\bigvee \Xi=\|\Upsilon\|_{C_{0}^{*}}$. Для обозначения интегралов в правых частях $(11)$ и (12) будем использовать в дальнейшем соответственно обозначения $\langle x, \xi\rangle=\langle x, \xi\rangle_{\rho}$ и $\langle x, d \Xi\rangle=\langle x, d \Xi\rangle_{\infty}$, так что

$$
\begin{aligned}
& \langle x, \xi\rangle=\langle x, \xi\rangle_{\rho}=\int x(t) \xi(t) d t, \quad 1 \leqslant \rho<\infty, \\
& \langle x, d \Xi\rangle=\langle x, d \Xi\rangle_{\infty}=\int x(t) d \Xi(t), \quad \rho=\infty .
\end{aligned}
$$

Пусть $\mathscr{L}_{\gamma}, 1 \leqslant \gamma \leqslant \infty$, при $\gamma=\infty$ есть пространство $\widetilde{L}_{\infty}$, при $1<\gamma<\infty-$ пространство $L_{\gamma}$, (а значит, пространство $\widetilde{L}_{\gamma}$ при $1<\gamma \leqslant \infty$ ) и, наконец, при $\gamma=1$ - пространство $M$. Для обозначения нормы элементов $x \in \mathscr{L}_{\gamma}$ будет использоваться обозначение $\|x\|_{\mathscr{L}_{\gamma}}$, а также обозначение $\|x\|_{\gamma}$; в частности, $\|\Xi\|_{1}=\bigvee \Xi, \Xi \in M=\mathscr{L}_{1}$. В этих обозначениях имеем $L_{\rho}^{*}=\mathscr{L}_{\rho^{\prime}}, 1 \leqslant \rho \leqslant \infty$.

Расширим область определения рассматриваемых здесь операторов. Пусть $\mathscr{M}=$ $\mathscr{M}\left(\mathbb{R}^{m}\right)$ есть пространство $\sigma$-аддитивных $\sigma$-конечных (знакопеременных) мер Лебега $\Xi$ на $\mathbb{R}^{m}$, для каждой из которых найдется показатель $\mu=\mu(\Xi) \leqslant 0$ со свойством

$$
\int\left(1+|t|^{2}\right)^{\mu} d|\Xi|(t)<\infty
$$

где $|\Xi|$ - модуль меры $\Xi$, т.е. (неотрицательная) мера, заданная соотношением $|\Xi|=$ $\Xi^{+}-\Xi^{-}$, и $\Xi^{+}, \Xi^{-}$есть неотрицательная и неположительная составляющие меры $\Xi$. В дальнейшем нам удобно будет иногда меру $\Xi \in \mathscr{M}$ обозначать также через $\xi=d \Xi$. Ясно, что $M \subset \mathscr{M} \subset \mathscr{S}^{\prime}$. Для локально абсолютно непрерывной меры $\Xi \in \mathscr{M}$ ее производная $\xi$ (относительно классической меры Лебега $d t$ ) принадлежит пространству $\Sigma$. Обратно, каждая функция $\xi \in \Sigma$ порождает естественньм образом меру $\Xi \in \mathscr{M}$. В этом смысле $\Sigma \subset \mathscr{M}$. Пусть $\Theta$ - любой из операторов $A, A^{*}, B, B^{*}, H, H^{*}$. Если функция $x \in \Sigma$ и мера $\Xi \in \mathscr{M}$ таковы, что для любой функции $\phi \in \mathscr{S}$ имеет место равенство

$$
\int x(t)\left(\Theta^{*} \phi\right)(t) d t=\int \phi(t) d \Xi(t),
$$

то будем считать, что $x$ принадлежит области определения $\mathscr{D}(\Theta)$ оператора $\Theta$ и мера $\Xi$ или, что то же самое, $\xi=d \Xi$ есть значение $\Theta x$ оператора $\Theta$ на функции $x$.

Для пары пространств $\mathscr{X}, \mathscr{Y}$, каждое из которых есть либо одно из определенных выше функциональных пространств $\Sigma, L_{\rho}, \widetilde{L}_{\rho}, C$, либо одно из пространств мер $\mathscr{M}, M$, обозначим через $\mathscr{D}(\mathscr{X}, \mathscr{Y} ; \Theta)$ множество элементов (т.е. функций или мер) $x \in \mathscr{X}$, таких, что значение $\Theta x$ оператора $\Theta$ на элементе $x$, определяемое по схеме Соболева, принадлежит $\mathscr{Y ~ . ~ Т а к и м ~ о б р а з о м , ~ т о л ь к о ~ ч т о ~ в в е д е н н о е ~ м н о ж е с т в о ~} \mathscr{D}(\Theta)$ есть $\mathscr{D}(\Sigma, \mathscr{M} ; \Theta)$. Кроме того, в этих обозначениях, к примеру, имеем $D(\Theta)=\mathscr{D}(\Sigma, \Sigma ; \Theta), W_{p}(\Theta)=\mathscr{D}\left(\Sigma, L_{p} ; \Theta\right)$, $W_{r, p}(\Theta)=\mathscr{D}\left(L_{r}, L_{p} ; \Theta\right)$.

Введем пространства

$$
\begin{aligned}
& \mathscr{W}_{r^{\prime}}\left(B^{*}\right)=\left\{\varphi \in \mathscr{D}\left(B^{*}\right): B^{*} \varphi \in \mathscr{L}_{r^{\prime}}\right\}=\mathscr{D}\left(\Sigma, \mathscr{L}_{r^{\prime}} ; B^{*}\right), \\
& \mathscr{W}_{q^{\prime}}\left(H^{*}\right)=\left\{\psi \in \mathscr{D}\left(H^{*}\right): H^{*} \psi \in \mathscr{L}_{q^{\prime}}\right\}=\mathscr{D}\left(\Sigma, \mathscr{L}_{q^{\prime}} ; H^{*}\right)
\end{aligned}
$$


и выделим в них классы

$$
\begin{gathered}
\mathscr{Q}_{r^{\prime}}\left(B^{*}\right)=\left\{\varphi \in \mathscr{W}_{r^{\prime}}\left(B^{*}\right):\left\|B^{*} \varphi\right\|_{r^{\prime}} \leqslant 1\right\}, \\
\mathscr{Q}_{2}=N \mathscr{Q}_{r^{\prime}}\left(B^{*}\right)=\left\{\varphi \in \mathscr{W}_{r^{\prime}}\left(B^{*}\right):\left\|B^{*} \varphi\right\|_{r^{\prime}} \leqslant N\right\}, \\
\mathscr{Q}_{1}=\mathscr{Q}_{q^{\prime}}\left(H^{*}\right)=\left\{\psi \in \mathscr{W}_{q^{\prime}}\left(H^{*}\right):\left\|H^{*} \psi\right\|_{q^{\prime}} \leqslant 1\right\} .
\end{gathered}
$$

Для элемента $\psi \in \mathscr{W}_{q^{\prime}}\left(H^{*}\right)$ положим

$$
\mathscr{F}\left(\psi, N \mathscr{Q}_{r^{\prime}}\left(B^{*}\right)\right)_{p^{\prime}}=\inf \left\{\|\psi-\varphi\|_{p^{\prime}}: \varphi \in N \mathscr{Q}_{r^{\prime}}\left(B^{*}\right)\right\}, \quad \psi \in \mathscr{W}_{q^{\prime}}\left(H^{*}\right) ;
$$

это есть наилучшее приближение в пространстве $\widetilde{L}_{p^{\prime}}$ функции $\psi \in \mathscr{W}_{q^{\prime}}\left(H^{*}\right)$ классом $\mathscr{Q}_{2}=N \mathscr{Q}_{r^{\prime}}\left(B^{*}\right)$. Тогда величина

$$
\mathscr{F}(N)=\mathscr{F}\left(\mathscr{Q}_{q^{\prime}}\left(H^{*}\right), N \mathscr{Q}_{r^{\prime}}\left(B^{*}\right)\right)_{p^{\prime}}=\sup \left\{\mathscr{F}\left(\psi, N \mathscr{Q}_{r^{\prime}}\left(B^{*}\right)\right)_{p^{\prime}}: \psi \in \mathscr{Q}_{q^{\prime}}\left(H^{*}\right)\right\}
$$

есть наилучшее приближение в $\widetilde{L}_{p^{\prime}}$ класса $\mathscr{Q}_{1}=\mathscr{Q}_{q^{\prime}}\left(H^{*}\right)$ классом $\mathscr{Q}_{2}=N \mathscr{Q}_{r^{\prime}}\left(B^{*}\right)$. Обозначим далее через $\mathscr{P}(N)=\mathscr{P}\left(\mathscr{Q}_{1}, \mathscr{Q}_{2}\right)=\mathscr{P}\left(\mathscr{Q}_{q^{\prime}}\left(H^{*}\right), N \mathscr{Q}_{r^{\prime}}\left(B^{*}\right)\right)$ множество линейных отображений класса $\mathscr{Q}_{1}$ в класс $\mathscr{Q}_{2}$, а точнее, множество линейных отображений $P$ пространства $\mathscr{W}_{q^{\prime}}\left(H^{*}\right)$ в пространство $\mathscr{W}_{r^{\prime}}\left(B^{*}\right)$ со свойством

$$
\left\|B^{*} P \psi\right\|_{r^{\prime}} \leqslant N\left\|H^{*} \psi\right\|_{q^{\prime}}, \quad \psi \in \mathscr{W}_{q^{\prime}}\left(H^{*}\right),
$$

или, что то же самое, $P \mathscr{Q}_{q^{\prime}}\left(H^{*}\right) \subset N \mathscr{Q}_{r^{\prime}}\left(B^{*}\right)$. Для оператора $P \in \mathscr{P}(N)$ положим

$$
\mathscr{J}(P)=\mathscr{G}\left(\mathscr{Q}_{q^{\prime}}\left(H^{*}\right) ; P\right)_{p^{\prime}}=\sup \left\{\|\psi-P \psi\|_{p^{\prime}}: \psi \in \mathscr{Q}_{q^{\prime}}\left(H^{*}\right)\right\} .
$$

Тогда величина

$\mathscr{G}(N)=\mathscr{G}\left(\mathscr{Q}_{1}, \mathscr{Q}_{2}\right)_{p^{\prime}}=\mathscr{G}\left(\mathscr{Q}_{q^{\prime}}\left(H^{*}\right), N \mathscr{Q}_{r^{\prime}}\left(B^{*}\right)\right)_{p^{\prime}}=\inf \left\{\mathscr{G}\left(\mathscr{Q}_{q^{\prime}}\left(H^{*}\right) ; P\right)_{p^{\prime}}: P \in \mathscr{P}(N)\right\}$

есть наилучшее линейное приближение в пространстве $\widetilde{L}_{p^{\prime}}$ класса $\mathscr{Q}_{1}=\mathscr{Q}_{q^{\prime}}\left(H^{*}\right)$ классом $\mathscr{Q}_{2}=N \mathscr{Q}_{r^{\prime}}\left(B^{*}\right)$. Вероятно, более естественно вьглядят задачи исследования величин $* \mathscr{F}(N)$ и $* \mathscr{G}(N)$ соответственно наилучшего и наилучшего линейного приближения в пространстве $\widetilde{L}_{p^{\prime}}$ класса $* \mathscr{Q}_{1}=\mathscr{Q}_{q^{\prime}}(H)$ классом $* \mathscr{Q}_{2}=N \mathscr{Q}_{r^{\prime}}(B)$. Однако, нетрудно убедиться, что

$$
* \mathscr{F}(N)=\mathscr{F}(N), \quad * \mathscr{G}(N)=\mathscr{G}(N),
$$

и при этом, именно задачи $\mathscr{F}(N)$ и $\mathscr{G}(N)$ естественньм образом возникнут в дальнейших наших рассуждениях.

Ниже при исследовании задач (в случае $p=\infty$ ) возникнет еще одно условие на оператор $B$. Область определения оператора $B$ можно расширить до множества $\mathscr{D}(\mathscr{M}, \mathscr{M} ; B)$. А именно, относительно мер $X \in \mathscr{M}$ и $\Xi \in \mathscr{M}$ будем считать, что $X$ принадлежит области определения $\mathscr{D}(\mathscr{M}, \mathscr{M} ; B)$ оператора $B$, и мера $\Xi$ есть значение $B X$ оператора $B$ на функции $X: \Xi=B X$, если для любой функции $\phi \in \mathscr{S}$ имеет место равенство

$$
\int\left(B^{*} \phi\right)(t) d X(t)=\int \phi(t) d \Xi(t) .
$$

Обозначим через $(\mathrm{M})$ условие, которое состоит в том, что любая мера $\Xi \in \mathscr{D}(\mathscr{M}, \mathscr{M} ; B)$ локально абсолютно непрерывна, и, как следствие, производная $d \Xi$ каждой такой меры относительно классической меры Лебега $d t$ принадлежит $\Sigma$, т.е. $\mathscr{D}(\mathscr{M}, \mathscr{M} ; B)=\mathscr{D}(\Sigma$, $\mathscr{M} ; B)$. Этим свойством обладает, к примеру, довольно широкий класс операторов дифференцирования с постоянными коэффициентами. Однако, в дальнейшем нам понадобится несколько более слабое по сравнению с (M) условие на оператор $B$ и параметр $\rho$, $1 \leqslant \rho \leqslant \infty$, которое мы обозначим через $(\mathrm{M}, \rho):$ любая мера $\Xi \in \mathscr{D}\left(\mathscr{M}, \mathscr{L}_{\rho} ; B\right)$ локально абсолютно непрерьвна, т.е. $\mathscr{D}\left(\mathscr{M}, \mathscr{L}_{\rho} ; B\right)=\mathscr{D}\left(\Sigma, \mathscr{L}_{\rho} ; B\right)$. 
2. Вспомогательные утверждения. Пространство

$$
W_{r, p}=W_{r, p}(B)=\left\{x \in L_{r} \cap \mathscr{D}(B): B x \in L_{p}\right\}
$$

является банаховым относительно нормы

$$
\|x\|_{W_{r, p}}=\|x\|_{r}+\|B x\|_{p},
$$

что легко следует из соотношения

$$
\int x B^{*} \phi d t=\int \phi B x d t, \quad \phi \in \mathscr{S}
$$

определяющего элемент $B x$, и предположения $B \mathscr{S} \subset \mathscr{S}$. Обозначим через $\stackrel{\circ}{W}_{r, p}(B)$ замькание множества $\mathscr{S}_{\text {в }} W_{r, p}(B)$. Ясно, что $\stackrel{\circ}{W}_{r, p}(B) \subset W_{r, p}(B)$; это вложение может быть строгим (см. пример в [10]). В дальнейшем для нас важным будет являться случай, когда множество $\mathscr{S}$ плотно в $W_{r, p}(B)$, т.е. имеет место свойство

$$
\stackrel{\circ}{W}_{r, p}(B)=W_{r, p}(B) .
$$

В настоящее время имеется много результатов, посвященных подобным вопросам (см. монографии [19]-[21] и обзор [5]). Следующее утверждение содержится в работах автора [12] и [10]. Для удобства формулировки этого утверждения и дальнейших ссылок введем условие на параметр $p$ и оператор $B$, которое будет обозначаться через $(B, p)$.

Условие $(B, p)$ состоит в том, что в случае $1<p \leqslant \infty$ оператор $B$ обладает свойством

$$
\left\{g \in C_{0}, B g=0\right\} \Longrightarrow g=0,
$$

а в случае $p=1-$ свойством

$$
\{g \in C, B g=0\} \Longrightarrow g=0 .
$$

Лемма 1. Если выполнено условие $(B, p)$, то мнохсество $\mathscr{S}$ плотно в $W_{r, p}(B)$, m.е. имеет место (16).

Как видно из следуюшего довольно простого утверждения, доказательство которого можно найти в $[12]$, условие $(B, p)$ обеспечивает также плотность множества $B \mathscr{S}$ в пространстве $L_{p}$.

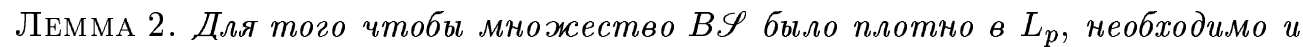
достаточно, чтобы оператор $B$ обладал свойством

$$
\left\{g \in L_{p^{\prime}} \cap C_{0}, B g=0\right\} \Longrightarrow g=0,
$$

если $p>1$, и свойством (17), если $p=1$.

Задачи (3) и (2) естественно изучать в том случае, когда выполнено следующее условие:

(Е) имеет место вложение

$$
W_{r, p}(B) \subset W_{r, q}(A),
$$

и, более того, существует (конечная) константа $K$ такая, что

$$
\|A x\|_{q} \leqslant K\left(\|x\|_{r}+\|B x\|_{p}\right), \quad x \in W_{r, p}(B) .
$$


В дальнейшем мы всегда будем предполагать, что это условие выполнено. Исследованию этого свойства посвящено много работ (см. монографии [19]-[21], работы [22], [23] и приведенную в них библиографию). Так известно [22], что при $m=1$, т.е. на оси (а также и на полуоси $[0, \infty))$ для операторов дифференцирования порядка $k$ и $n, 0 \leqslant k<n$, за исключением некоторых вырожденных значений параметров, неравенство (18) или, что то же самое, неравенство (6) с конечной константой имеет место в том и только том случае, если вьполняется условие

$$
\frac{n-k}{r}+\frac{k}{p} \geqslant \frac{n}{q}
$$

Лемма 3. Допустим, что выполнено условие факторизации $(\Phi)$, условие $(B, p)$ и условие (Е). Тогда если для пары функиий $\psi \in \mathscr{W}_{q^{\prime}}\left(H^{*}\right), \varphi \in \mathscr{W}_{r^{\prime}}\left(B^{*}\right)$ разность $\chi=\psi-\varphi$ принадлежит пространству $\widetilde{L}_{p^{\prime}}$, то для любой функиии $x \in W_{r, p}(B)$ справедливо представление

$$
\left\langle A x, H^{*} \psi\right\rangle_{q}-\left\langle x, B^{*} \varphi\right\rangle_{r}=\int(B x)(t) \chi(t) d t .
$$

ДокАЗАТЕЛЬСтво. Пусть вначале $x \in \mathscr{S}$, а значит, и $A x \in \mathscr{S}$. Исходя из определения функции $H^{*} \psi$ в силу предположения $(\Phi)$ получаем

$$
\left\langle A x, H^{*} \psi\right\rangle_{q}=\int(H A x)(t) \psi(t) d t=\int(B x)(t) \psi(t) d t .
$$

Кроме того, имеем

$$
\left\langle x, B^{*} \varphi\right\rangle_{r}=\int(B x)(t) \varphi(t) d t
$$

Эти соотношения дают представление (19) на функциях $x \in \mathscr{S}$.

Пусть теперь $x \in W_{r, p}(B)$. В силу предположений $(B, p)$ существует последовательность $\left\{x_{n}\right\}_{n=1}^{\infty} \subset \mathscr{S}$ такая, что $\left\|x-x_{n}\right\|_{W_{r, p}} \rightarrow 0$ при $n \rightarrow \infty$, т.е. $\left\|x-x_{n}\right\|_{r} \rightarrow 0$ и $\left\|B x-B x_{n}\right\|_{p} \rightarrow 0$ при $n \rightarrow \infty$. Согласно условию (Е) будет иметь место также свойство $\left\|A x-A x_{n}\right\|_{q} \rightarrow 0$ при $n \rightarrow \infty$. Записав (19) для функций $x_{n}$ и перейдя в полученном соотношении к пределу при $n \rightarrow \infty$, получим (19) для функции $x$. Лемма доказана.

Для функции $\psi \in \mathscr{W}_{q^{\prime}}\left(H^{*}\right)$ и линейного ограниченного функционала $\Upsilon \in L_{r}^{*}$ рассмотрим величину

$$
\nu(\psi, \Upsilon)=\sup \left\{\left\langle A x, H^{*} \psi\right\rangle_{q}-\Upsilon x: x \in Q_{r, p}(B)\right\}
$$

Лемма 4. Допустим, что выполнено условие факторизации $(\Phi)$, условие $(B, p)$, условие (Е) и в случае $p=\infty$ - условие $\left(\mathrm{M}, r^{\prime}\right)$. Тогда если функиия $\psi \in \mathscr{W}_{q^{\prime}}\left(H^{*}\right) u$ функиионал $\Upsilon \in L_{r}^{*}$, представленный в виде (11) при $1 \leqslant r<\infty u$ (12) при $r=\infty$, таковы, что $\nu(\psi, \Upsilon)<\infty$, то существует функиия

$$
\varphi \in \mathscr{W}_{r^{\prime}}\left(B^{*}\right)
$$

со свойствами

$$
\begin{gathered}
B^{*} \varphi= \begin{cases}\xi & \text { npu } 1 \leqslant r<\infty \\
d \Xi & \text { npu } r=\infty\end{cases} \\
\|\psi-\varphi\|_{p^{\prime}}=\nu(\psi, \Upsilon)
\end{gathered}
$$

и для любой функиии $x \in W_{r, p}(B)$ имеет место представление

$$
\left\langle A x, H^{*} \psi\right\rangle_{q}-\Upsilon x=\langle B x, \psi-\varphi\rangle_{p}
$$


ДокАЗАТЕЛЬСТво. Обозначим через $\mathscr{S}_{p}(B)$ множество функций $x \in \mathscr{S}$ с $\|B x\|_{p} \leqslant 1$ и положим

$$
\mu(\psi, \Upsilon)=\sup \left\{\left\langle A x, H^{*} \psi\right\rangle_{q}-\Upsilon x: x \in \mathscr{S}_{p}(B)\right\} .
$$

Поскольку $\mathscr{S}_{p}(B) \subset Q_{r, p}(B)$, то

$$
\mu(\psi, \Upsilon) \leqslant \nu(\psi, \Upsilon)<\infty
$$

Покажем, что существует функция $\varphi \in \mathscr{W}_{r^{\prime}}\left(B^{*}\right)$ со свойством $(22)$ такая, что для функций $x \in \mathscr{S}$ имеет место представление $(24)$, и вьполняется равенство

$$
\|\psi-\varphi\|_{p^{\prime}}=\mu(\psi, \Upsilon)
$$

аналогичное (23); этот факт мы будем назьвать в этом доказательстве утверждением леммы на множестве функций $\mathscr{S}$.

Применяя известные соображения Сарда (см., например, лемму 2 и ее применение в работе [24]), заключаем, что на множестве $\mathscr{S}$ имеет место представление

$$
\left\langle A x, H^{*} \psi\right\rangle_{q}-\Upsilon x=\Pi B x, \quad x \in \mathscr{S},
$$

где П - линейньй функционал на линейном пространстве $Y=B \mathscr{S}$. По предположению $Y=B \mathscr{S} \subset \mathscr{S}$ и, тем более, $Y \subset L_{p}$; наделим $Y$ нормой пространства $L_{p}$. Имеем $\|\Pi\|_{Y^{*}}=\mu(\psi, \Upsilon)$. Продолжим П линейно с сохранением нормы с $Y$ на все пространство $L_{p}$. Для функционала П на $L_{p}$ в зависимости от значения $p$ будет иметь место представление

$$
\Pi y=\langle y, \chi\rangle_{p}, \quad y \in L_{p},
$$

где

$$
\begin{gathered}
\chi \in \widetilde{L}_{p^{\prime}}, \quad \mu(\psi, \Upsilon)=\|\chi\|_{p^{\prime}} \quad \text { при } 1 \leqslant p<\infty, \\
\chi=d X, \quad X \in M, \quad \mu(\psi, \Upsilon)=\bigvee X \quad \text { при } \quad p=\infty .
\end{gathered}
$$

Поскольку $\psi \in \mathscr{W}_{q^{\prime}}\left(H^{*}\right)$ и $x \in \mathscr{S}$, то

$$
\left\langle A x, H^{*} \psi\right\rangle_{q}=\int(H A x)(t) \psi(t) d t=\int(B x)(t) \psi(t) d t .
$$

Дальнейшие рассуждения будут проведены в зависимости от значений параметров $r, p$. Рассмотрим вначале случай $1 \leqslant r, p<\infty$. Из $(26),(30),(27),(28)$ и (11) следует соотношение

$$
\Upsilon x=\int x(t) \xi(t) d t=\int(\psi(t)-\chi(t)) B x(t) d t, \quad x \in \mathscr{S}
$$

это соотношение означает, что разность $\varphi=\psi-\chi$ принадлежит области определения $\mathscr{D}\left(B^{*}\right)$ оператора $B^{*}$ и $\xi=B^{*} \varphi$, а следовательно, $\varphi \in \mathscr{W}_{r^{\prime}}\left(B^{*}\right)$. При этом в силу (28)

$$
\|\psi-\varphi\|_{p^{\prime}}=\|\chi\|_{p^{\prime}}=\mu(\psi, \Upsilon) .
$$

Соотношение (26) совпадает с (24) для $x \in \mathscr{S}$. Тем самым, мы проверили, что в случае $1 \leqslant r, p<\infty$ утверждение леммы на классе функций $\mathscr{S}$ вьполняется. 
Пусть теперь $r=\infty, 1 \leqslant p<\infty$. В этом случае $(26),(30),(27),(28)$ и (12) дают соотношение

$$
\Upsilon x=\int x(t) d \Xi(t)=\int(\psi(t)-\chi(t)) B x(t) d t, \quad x \in \mathscr{S},
$$

которое означает, что разность $\varphi=\psi-\chi$ принадлежит области определения $\mathscr{D}\left(B^{*}\right)$ оператора $B^{*}$ и $d \Xi=B^{*} \varphi$. Отсюда, как и вьше, заключаем, что и в случае $r=\infty$, $1 \leqslant p<\infty$ имеет место утверждение леммы на функциях $x \in \mathscr{S}$.

Нам осталось рассмотреть значения параметров $p=\infty, 1 \leqslant r \leqslant \infty$. Обозначим при $1 \leqslant r<\infty$ через $\Xi$ локально абсолютно непрерывную меру, производная которой есть функция $\xi$ из представления (11) функционала $\Upsilon$. Тогда (в рассматриваемом случае $p=\infty, 1 \leqslant r \leqslant \infty)$ соотношения $(26),(30),(27),(29),(11)$ и (12) дают представление

$$
\Upsilon x=\int x(t) d \Xi(t)=\int B x(t) d(\Psi(t)-X(t)), \quad x \in \mathscr{S},
$$

где $\Psi$ есть локально абсолютно непрерьвная мера, производной которой является функция $\psi$. Соотношение (31) означает, что мера $\Phi=\Psi-X$ принадлежит множеству $\mathscr{D}\left(\mathscr{M}, L_{r}^{*} ; B^{*}\right)$ и $\Xi=B^{*} \Phi$. В силу предположения $\left(\mathrm{M}, r^{\prime}\right)$ мера $\Phi$ локально абсолютно непрерьвна; обозначим ее производную через $\varphi$. Мера $X=\Psi-\Phi$ как разность локально абсолютно непрерьвных мер будет также локально абсолютно непрерывна. В силу (29) производная $\chi$ меры $X$ суммируема и

$$
\mu(\psi, \Upsilon)=\bigvee X=\|\chi\|_{1}=\|\psi-\varphi\|_{1}
$$

Таким образом, утверждения леммы на множестве $\mathscr{S}$ справедливы для всех значений параметров. В условиях леммы 4 выполняются и условия леммы 3 на параметры и операторы. Кроме того, функции $\psi, \varphi$ и $\chi=\psi-\varphi$ также удовлетворяют условиям леммы 3 . Согласно лемме 3 на множестве $W_{r, p}(B)$ имеет место представление $(24)$. Из этого представления следует, что

$$
\nu(\psi, \Upsilon)=\sup \left\{\left\langle A x, H^{*} \psi\right\rangle_{q}-\Upsilon x: x \in Q_{r, p}(B)\right\} \leqslant\|\chi\|_{p^{\prime}}=\|\psi-\varphi\|_{p^{\prime}}=\mu(\psi, \Upsilon) .
$$

В силу (25) это влечет свойство (23).

Убедимся, что в условиях леммы для любой функции $\psi \in \mathscr{W}_{q^{\prime}}\left(H^{*}\right)$ функция $\varphi$ со свойствами (21)-(23) единственная. Предположим, что для некоторой функции $\psi$ нашлись две функции $\varphi_{1}, \varphi_{2}$ со свойствами (21)-(23). Тогда их разность $\varphi=\varphi_{2}-\varphi_{1}$ принадлежит пространству $\mathscr{D}\left(\widetilde{L}_{p^{\prime}}, \mathscr{L}_{r^{\prime}} ; B^{*}\right)$ и является решением однородного уравнения $B^{*} \varphi=0$. В силу определения (1) отсюда следует, что

$$
\int(B \zeta)(t) \varphi(t) d t=0, \quad \zeta \in \mathscr{S} .
$$

Согласно лемме 2 условие $(B, p)$ влечет плотность множества $B \mathscr{S}$ в пространстве $L_{p}$. Поэтому из (32) следует, что $\varphi=0$. Лемма 4 полностью доказана.

Для функции $\psi \in \mathscr{W}_{q^{\prime}}\left(H^{*}\right)$ положим

$$
\sigma(\psi, N)=\inf \left\{\nu(\psi, \Upsilon): \Upsilon \in L_{r}^{*},\|\Upsilon\| \leqslant N\right\}
$$

где величина $\nu(\psi, \Upsilon)$ определена формулой $(20)$. Сейчас мы покажем, что $\sigma(\psi, N)$ совпадает с величиной

$$
\left.\mathscr{F}(\psi, N)=\mathscr{F}\left(\psi, N \mathscr{Q}_{r^{\prime}}\left(B^{*}\right)\right)_{p^{\prime}}=\inf \{\| \psi-\varphi) \|_{p^{\prime}}: \varphi \in N \mathscr{Q}_{r^{\prime}}\left(B^{*}\right)\right\} .
$$


СлЕДСТВИЕ 1. Пусть выполнены условия леммы 4. Тогда для любой функиии $\psi \in \mathscr{W}_{q^{\prime}}\left(B^{*}\right)$ имеет место равенство

$$
\sigma(\psi, N)=\mathscr{F}\left(\psi, N \mathscr{Q}_{r^{\prime}}\left(B^{*}\right)\right)_{p^{\prime}}
$$

Действительно, неравенство $\sigma(\psi, N) \leqslant \mathscr{F}(\psi, N)$ вытекает из леммы 3. Обратное неравенство $\mathscr{F}(\psi, N) \leqslant \sigma(\psi, N)$ следует из леммы 4 .

ЗАмЕчАниЕ 1. Как видно из доказательства лемм 3,4 и следствия 1 , кроме равенства (34) можно утверждать, что каждому экстремальному функционалу $\Upsilon$ задачи (33) соответствует функция $\varphi \in N \mathscr{Q}_{r^{\prime}}\left(B^{*}\right)$, для которой $\|\psi-\varphi\|_{p^{\prime}}=\mathscr{F}(\psi, N)$, и, обратно, каждая такая функция порождает экстремальный функционал.

\section{3. Двойственность задач о вычислении модуля непрерывности оператора} и наилучшего приближения класса классом. В этом пункте мы будем использовать следующий известный результат В.Н. Габушина [25] о наилучшем приближении линейных неограниченных функционалов линейными ограниченными.

Лемма 5. Пусть $X$ есть банахово пространство, $\Lambda$ - линейный (не обязательно ограниченный) функционал на $X, \mathscr{K}$ - чентрально симметричное выпуклое множество, принадлежсащее области определения функщионала $\Lambda$. Тогда при любом $N>0$ для величинь

$$
e(N)=\inf _{\|\Upsilon\|_{X^{*}} \leqslant N} \sup _{x \in K}(\Lambda x-\Upsilon x)
$$

имеет место равенство

$$
e(N)=\sup _{x \in \mathscr{K}}\left(\Lambda x-N\|x\|_{X}\right)
$$

В приводимых ниже утверждениях на оператор $H$ будет наложено еше одно ограничение $(H, \rho)$ : множество $H W_{\rho}(H)$ плотно в пространстве $L_{\rho}$.

Ясно, что условие $(H, \rho)$ будет вьполнено, если множество $H \mathscr{S}$ будет плотно в $L_{\rho}$; поэтому лемма 3 дает достаточные условия выполнения свойства $(H, \rho)$.

ТЕоремА 1. Пусть выполнены условие факторизации $(\Phi)$, условие $(B, p)$, условие $(\mathrm{E})$, условие $\left(H, q^{\prime}\right)$ и в случае $p=\infty$ - условие $\left(\mathrm{M}, r^{\prime}\right)$. Тогда при любом $N>0$ задачи (3) и (14) связаны равенством $R(N)=\mathscr{F}(N)$.

ДоКАЗАТЕЛЬСТВо. Положим

$$
\sigma(N)=\sup \left\{\sigma(\psi, N): \psi \in \mathscr{Q}_{q^{\prime}}\left(H^{*}\right)\right\}
$$

где величина $\sigma(\psi, N)$ определена формулами $(33),(20)$. В силу (34) имеем

$$
\begin{aligned}
\sigma(N) & =\sup \left\{\sigma(\psi, N): \psi \in \mathscr{Q}_{q^{\prime}}\left(H^{*}\right)\right\} \\
& =\sup \left\{\mathscr{F}\left(\psi, N \mathscr{Q}_{r^{\prime}}\left(B^{*}\right)\right)_{p^{\prime}}: \psi \in \mathscr{Q}_{q^{\prime}}\left(H^{*}\right)\right\}=\mathscr{F}(N) .
\end{aligned}
$$

С другой стороны, согласно лемме 5 справедливо равенство

$$
\sigma(\psi, N)=\sup \left\{\left\langle A x, H^{*} \psi\right\rangle_{q}-N\|x\|_{r}: x \in Q_{r, p}(B)\right\} .
$$


Отсюда

$$
\sigma(N)=\sup _{x \in Q_{r, p}(B)} \sup _{\psi \in \mathscr{Q}_{q^{\prime}}\left(H^{*}\right)}\left\{\left\langle A x, H^{*} \psi\right\rangle_{q}-N\|x\|_{r}\right\} .
$$

Далее, условие $\left(H, q^{\prime}\right)$ приводит к тому, что

$$
\sup \left\{\left\langle A x, H^{*} \psi\right\rangle_{q}: \psi \in \mathscr{Q}_{q^{\prime}}\left(H^{*}\right)\right\}=\|A x\|_{q},
$$

а это дает, окончательно, соотношение

$$
\sigma(N)=\sup \left\{\|A x\|_{q}-N\|x\|_{r}: x \in Q_{r, p}(B)\right\}=R(N) .
$$

Теорема 1 доказана.

4. Двойственность задачи Стечкина о приближении неограниченного оператора ограниченными и задачи о линейном приближении класса класcom.

Лемма 6. Для любых значений параметров $p, q, r(1 \leqslant p, q, r \leqslant \infty)$, если выполнено условие факторизации $(\Phi)$, условие $(B, p)$, условие $(\mathrm{E})$ и в случае $p=\infty$ условие (M, $\left.r^{\prime}\right)$, то задачи (3) и (14) связаны неравенством

$$
\mathscr{G}(N) \leqslant E(N) .
$$

ДокАЗАтЕльСтво. Достаточно рассмотреть случай $E(N)<\infty$. Допустим, что для линейного ограниченного оператора $T \in \mathscr{B}_{r}^{q}(N)$ величина $U(T)$ конечна. Функции $\psi \in \mathscr{W}_{q^{\prime}}\left(H^{*}\right)$ сопоставим функционал $\Upsilon=\Upsilon_{\psi}$ по формуле

$$
\Upsilon x=\Upsilon_{\psi} x=\left\langle T x, H^{*} \psi\right\rangle_{q} .
$$

Видно, что $\Upsilon_{\psi}$ есть линейный ограниченный функционал на $L_{r}$, т.е. $\Upsilon_{\psi} \in L_{r}^{*}$ и к тому же

$$
\begin{gathered}
\left\|\Upsilon_{\psi}\right\|_{L_{r}^{*}} \leqslant\left\|H^{*} \psi\right\|_{q^{\prime}} N \\
\sup \left\{\left\langle A x, H^{*} \psi\right\rangle_{q}-\Upsilon_{\psi} x: x \in Q_{r, p}\right\} \\
=\sup \left\{\left\langle A x-T x, H^{*} \psi\right\rangle_{q}: x \in Q_{r, p}\right\} \\
\leqslant U(T)\left\|H^{*} \psi\right\|_{q^{\prime}} .
\end{gathered}
$$

Согласно лемме 4 сушествует функция $\varphi \in \mathscr{W}_{r^{\prime}}\left(B^{*}\right)$ со свойствами $(11),(12),(22),(23)$. Отсюда будет следовать, что она обладает свойствами

$$
\|\psi-\varphi\|_{p^{\prime}} \leqslant U(T)\left\|H^{*} \psi\right\|_{q^{\prime}}, \quad\left\|B^{*} \varphi\right\|_{r^{\prime}} \leqslant N\left\|H^{*} \psi\right\|_{q^{\prime}} .
$$

$\Phi$ ункция $\varphi$ функцией $\psi$ определяется однозначно и, таким образом, $\varphi=P \psi$, где $P$ есть некоторое (однозначное) отображение пространства $\mathscr{W}_{q^{\prime}}\left(H^{*}\right)$ в пространство $\mathscr{W}_{r^{\prime}}\left(B^{*}\right)$. Как следствие (36) для величины

$$
\mathscr{J}(P)=\mathscr{G}\left(\mathscr{Q}_{q^{\prime}}\left(H^{*}\right) ; P\right)_{p^{\prime}}=\sup \left\{\|\psi-P \psi\|_{p^{\prime}}: \psi \in \mathscr{Q}_{q^{\prime}}\left(H^{*}\right)\right\}
$$


имеет место оценка

$$
\mathscr{J}(P) \leqslant U(T) .
$$

Убедимся, что $P$ является линейным отображением пространства $\mathscr{W}_{q^{\prime}}\left(H^{*}\right)$ в пространство $\mathscr{W}_{r^{\prime}}\left(B^{*}\right)$. Пусть $\psi_{1}, \psi_{2}$ есть две функции из $\mathscr{W}_{q^{\prime}}\left(H^{*}\right)$ и $c_{1}, c_{2}$ - две вещественные константы. Введем функцию $\psi=c_{1} \psi_{1}+c_{2} \psi_{2}$; эта функция также принадлежит пространству $\mathscr{W}_{q^{\prime}}\left(H^{*}\right)$. Положим $\varphi_{1}=P \psi_{1}, \varphi_{2}=P \psi_{2}, \varphi=P \psi, \widetilde{\varphi}=\varphi-\left(c_{1} \varphi_{1}+c_{2} \varphi_{2}\right)=P \psi-$ $\left(c_{1} P \psi_{1}+c_{2} P \psi_{2}\right)$. Функция $\widetilde{\varphi}$ принадлежит пространству $\widetilde{L}_{p^{\prime}}$ и удовлетворяет уравнению $B^{*} \widetilde{\varphi}=0$. Отсюда, также как при доказательстве леммы 4 , заключаем, что $\widetilde{\varphi}=0$. А этот факт означает, что оператор $P$ является линейным и, значит, $P \in \mathscr{P}(N)$. Из $(37)$ следует теперь неравенство (35). Лемма доказана.

В следующем утверждении будет доказано неравенство, обратное (35), хотя и при более сильных в сравнении с леммой 6 ограничениях.

Лемма 7. Допустим, что $1<q<\infty, 1 \leqslant p, r \leqslant \infty$, выполнено условие факторизачии $(\Phi)$, условие $(B, p)$, условие $(\mathrm{E})$, условие $\left(H, q^{\prime}\right)$ и в случае $p=\infty-$ условие $\left(\mathrm{M}, r^{\prime}\right)$. Тогда при любом $N>0$ имеет место неравенство

$$
E(N) \leqslant \mathscr{G}(N)
$$

ДоказАтЕЛьство. Допустим, что $\mathscr{G}(N)<\infty$, и оператор $P \in \mathscr{P}(N)$ обладает свойством $\mathscr{J}(P)<\infty$. Тот факт, что $P \in \mathscr{P}(N)$ означает, что $P$ есть линейное отображение пространства $\mathscr{W}_{q^{\prime}}\left(H^{*}\right)$ в пространство $\mathscr{W}_{r^{\prime}}\left(B^{*}\right)$ со свойством

$$
\left\|B^{*} P \psi\right\|_{r^{\prime}} \leqslant N\left\|H^{*} \psi\right\|_{q^{\prime}}, \quad \psi \in \mathscr{W}_{q^{\prime}}\left(H^{*}\right) .
$$

Отсюда следует, что функция $\varphi=B^{*} P \psi$ однозначно определяется функцией $H^{*} \psi$, $\psi \in \mathscr{W}_{q^{\prime}}\left(H^{*}\right)$, т.е. $\varphi=B^{*} P \psi$ есть значение некоторого (однозначного) отображения $\mathscr{T}$ на функции $H^{*} \psi$. Нетрудно понять, что $\mathscr{T}$ есть линейный ограниченньй оператор из пространства $\mathscr{H}=H^{2} \mathscr{W}_{q^{\prime}}\left(H^{*}\right)=\left\{H^{*} \psi: \psi \in \mathscr{W}_{q^{\prime}}\left(H^{*}\right)\right\}$, наделенного нормой про-

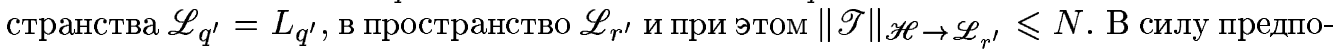
ложения $\left(H, q^{\prime}\right)$ множество $\mathscr{H}$ плотно в пространстве $L_{q^{\prime}}$. Поэтому оператор $\mathscr{T}$ можно продолжить с $\mathscr{H}$ на $L_{q^{\prime}}$ по непрерывности (и, значит, с сохранением нормы). Обозначим это продолжение тем же символом $\mathscr{T}$.

Пусть $T=\mathscr{T}^{*}$ есть оператор, сопряженньй оператору $\mathscr{T}$. Тогда $T$ есть линейный ограниченньй оператор из пространства $\mathscr{L}_{r^{\prime}}^{*}$ в пространство $L_{q^{\prime}}^{*}=L_{q}$ (напомним предположение $1<q<\infty)$. Мы будем рассматривать оператор $T$ лишь на подпространстве $L_{r} \subset \mathscr{L}_{r^{\prime}}^{*}$. Для нормы оператора $T$ справедлива оценка

$$
\|T\|=\|T\|_{L_{r} \rightarrow L_{q}} \leqslant\|T\|_{\mathscr{L}_{r^{\prime}}^{*} \rightarrow L_{q}}=\|\mathscr{T}\|_{L_{q^{\prime}} \rightarrow \mathscr{L}_{r^{\prime}}} \leqslant N .
$$

Далее, в силу определения оператора $T$ для любых функций $\psi \in \mathscr{W}_{q^{\prime}}\left(H^{*}\right)$ и $x \in L_{r}$ имеем

$$
\left\langle x, B^{*} P \psi\right\rangle_{r}=\left\langle x, \mathscr{T} H^{*} \psi\right\rangle_{r}=\left\langle T x, H^{*} \psi\right\rangle_{q},
$$

а значит, если $\psi \in \mathscr{W}_{q^{\prime}}\left(H^{*}\right)$ и $x \in W_{r, p}$, то

$$
\left\langle A x-T x, H^{*} \psi\right\rangle_{q}=\left\langle A x, H^{*} \psi\right\rangle_{q}-\left\langle T x, H^{*} \psi\right\rangle_{q}=\left\langle A x, H^{*} \psi\right\rangle_{q}-\left\langle x, B^{*} P \psi\right\rangle_{r} .
$$


Пара функций $\psi$ и $\varphi=B^{*} P \psi$ удовлетворяют условиям леммы 3. В силу этой леммы для функций $x \in W_{r, p}$ справедливо соотношение

$$
\left\langle A x-T x, H^{*} \psi\right\rangle_{q}=\langle B x, \psi-\varphi\rangle_{p}
$$

Отсюда следует, что

$$
\left|\left\langle A x-T x, H^{*} \psi\right\rangle_{q}\right| \leqslant\|B x\|_{p}\|\psi-\varphi\|_{p^{\prime}}
$$

Используя предположение $\left(H, q^{\prime}\right)$, заключаем, что имеет место оценка $U(T) \leqslant \mathscr{J}(P)$. А это оценка влечет неравенство (38). Лемма доказана.

Объединяя последние две леммы, получаем следующее утверждение.

Теорема 2. Допустим, ито $1<q<\infty, 1 \leqslant p, r \leqslant \infty$, выполнено условие факторизачии $(\Phi)$, условие $(B, p)$, условие $(\mathrm{E})$, условие $\left(H, q^{\prime}\right)$ и в случае $p=\infty-$ условие $\left(\mathrm{M}, r^{\prime}\right)$. Тогда задачи (3) и (14) связаны равенством $E(N)=\mathscr{G}(N)$.

ЗАмЕчАниЕ 2. При доказательстве последней теоремы, а точнее, в доказательствах двух последних лемм (по крайней мере в случае $1<q<\infty$ ) между множествами операторов $P \in \mathscr{P}(N)$ и $T \in \mathscr{B}_{r}^{q}(N)$, для которых соответствующие уклонения $\mathscr{J}(P)$ и $U(T)$ конечны, установлено взаимно однозначное соответствие: $B^{*}(P \psi)=\mathscr{T} H^{*} \psi$, где $\mathscr{T}$ - оператор, сопряженньй для оператора $T$; при этом $U(T)=\mathscr{J}(P)$. Отсюда следует, что если один из операторов $P$ или $T$ является экстремальным, то экстремальньп будет и второй (в соответствующей задаче).

5. Связь задач в случае $q=\infty$. Если элемент $\theta \in \mathscr{S}^{\prime}$ таков, что $\theta * \phi \in \mathscr{S}$ или, то же самое, $\check{\theta} * \phi \in \mathscr{S}$ для любой функции $\phi \in \mathscr{S}$, то можно говорить о свертке $\zeta * \theta$ элементов $\zeta \in \mathscr{S}^{\prime}$ и $\theta$, определив ее соотношением $\langle\zeta * \theta, \phi\rangle=\langle\zeta, \check{\theta} * \phi\rangle, \phi \in \mathscr{S}$. Напомним, что по предположению этим свойством обладают элементы $a, b, h \in \mathscr{S}^{\prime}$, определяющие операторы $A, B, H$ и им сопряженные операторы $A^{*}, B^{*}, H^{*}$.

Допустим, что существует фундаментальная функция $\vartheta$ оператора $H^{*}$, т.е. существует элемент $\vartheta \in \mathscr{S}^{\prime}$, такой, что свертка $\vartheta * h$ есть $\delta$-функция. В этом случае для любой функции $\phi \in \mathscr{S}$ имеем $\vartheta * b * \phi=\vartheta * h * a * \phi=\vartheta * h * A^{*} \phi=A^{*} \phi=a * \phi$, а значит, справедливо представление $a=\vartheta * b$. К примеру, если существует фундаментальная функция $\varkappa$ оператора $B^{*}$, то перечисленными свойствами обладает функция $\vartheta=a * \varkappa=A^{*} \varkappa$.

Предположим, что, к тому же, функция $\vartheta$ является классической функцией, а точнее, $\vartheta \in \Sigma$. Тогда, очевидно, имеем $\vartheta \in \mathscr{W}_{1}\left(H^{*}\right)$ и $H^{*} \vartheta$ есть атомная мера, сосредоточенная в точке $t=0$, вариация которой равна 1 ; таким образом, $\vartheta \in \mathscr{Q}_{1}\left(H^{*}\right)$. Рассмотрим величину (см. обозначение (13))

$$
\varepsilon(N)=\varepsilon(\vartheta ; r, p ; N)=\inf \left\{\|\vartheta-\varphi\|_{\widetilde{L}_{p^{\prime}}}: \varphi \in \mathscr{Q}_{r^{\prime}}\left(B^{*}\right)\right\}=\mathscr{F}\left(\vartheta, N \mathscr{Q}_{r^{\prime}}\left(B^{*}\right)\right)_{p^{\prime}}
$$

это есть наилучшее приближение в $\widetilde{L}_{p^{\prime}}$ функции $\vartheta$ множеством $N \mathscr{Q}_{r^{\prime}}\left(B^{*}\right)$. Величина, более общая, чем (39), в связи с задачей (2) изучалась в работе автора [12]; в теореме 2 работы [12] содержится, в частности, следующее утверждение. 
ЛЕмма 8. Если $q=\infty, 1 \leqslant p, r \leqslant \infty$, выполнено условие факторизачии $(\Phi)$ и условие $(B, p)$, то имеет место равенство

$$
E(N)=\varepsilon(N) .
$$

Следующее утверждение дополняет теоремы 1 и 2 в случае $q=\infty$. Последнее равенство в $(41)$ ниже означает, что функция $\vartheta \in \mathscr{Q}_{q^{\prime}}\left(H^{*}\right)$ является, в данной ситуации, максимальной в задаче (14), т.е. на ней в (14) достигается верхняя грань. Первое равенство в (41) есть результат В. Н. Габушина [25] и является следствием приведенной вьше леммы 5 .

ТЕорема 3. Если $q=\infty, 1 \leqslant p, r \leqslant \infty$, выполнено условие факторизачии $(\Phi)$, условие $(B, p)$, условие $(\mathrm{E})$ и в случае $p=\infty$ - условие $\left(\mathrm{M}, r^{\prime}\right)$, то имеют место равенства

$$
E(N)=R(N)=\mathscr{G}(N)=\mathscr{F}(N)=\varepsilon(N) .
$$

ДокАЗАТЕЛЬСТВо. Поскольку $\vartheta \in \mathscr{Q}_{q^{\prime}}\left(H^{*}\right)$, исходя из определений $(14),(15)$, используя неравенства (35) и равенство (40), будем иметь следующую цепочку соотношений:

$$
\varepsilon(N) \leqslant \mathscr{F}(N) \leqslant \mathscr{G}(N) \leqslant E(N)=\varepsilon(N) .
$$

А это влечет все утверждения теоремы 3 .

6. Заключительные замечания. Рассматриваемое в данной работе множество неограниченных линейных операторов $A$ и $B$ типа свертки содержит важньй класс дифференциальных операторов с постоянными коэффициентами. В настоящее время наиболее полно задачи (2), (3), (13), (15) изучены для операторов дифференцирования $A x=x^{(k)}, B x=x^{(n)}, 0 \leqslant k<n$, в пространствах функций одного переменного на оси (и полуоси); для функций многих переменных они изучены в существенно меньшей степени (см. библиографию в [4], [5]). Безусловно, в пространствах функций многих переменных все четыре задачи представляют большой интерес в случае, когда $A$ и $B$ есть степени (итерации)

$$
A=\Delta^{k}, \quad B=\Delta^{n}, \quad 0 \leqslant k<n,
$$

оператора Лапласа

$$
\Delta x=\sum_{j=1}^{m} \frac{\partial^{2} x}{\partial t_{j}^{2}} .
$$

Имеется ряд случаев, когда обсуждаемые в этой работе задачи для классов функций многих переменных могут быть решены точно. Так, без труда переносятся на многомерный случай одномерные результаты работ Субботина, Тайкова [16] для значений параметров $p=q=r=2$ и Тайкова [26] для $q=\infty, p=r=2$, в которых исследованы задачи (5), неравенство (6) (в работе [26]), а также соответствующие задачи приближения одного класса дифференцируемых функций другим классом более гладких функций. В работах [9], [10], [12] имеется ряд точных многомерных результатов, относящихся к задаче (2), полученных с помощью соображений инвариантности. В последнее время появился ряд интересных результатов о точном решении задач, близких к задачам (2), (3), на классах функций многих переменных, в которых в полной мере проявляется многомерность задач (см. работы [5], [27], [28] и содержащуюся там библиографию). Полученные в данной работе результаты позволяют в ряде новых случаев получить решение задач (13) и (15) как следствие соответствуюших результатов для задач $(2),(3)$. 


\section{СПИСОК ЦИТИРОВАННОЙ ЛИТЕРАТУРЫ}

[1] Стечкин С. Б. Наилучшее приближение линейных операторов // Матем. заметки. 1967. T. 1. №2. C. $137-148$.

[2] Арестов В. В. О некоторых экстремальных задачах для дифференцируемых функций одной переменной // Тр. МИАН. 1975. Т. 138. С. 3-28.

[3] Клоц Б. Е. Приближения дифференцируемых функций функциями большей гладкости // Матем. заметки. 1977. Т. 21. № 1. С. 21-32.

[4] Арестов В. В., Габушин В.Н. Наилучшее приближение неограниченных операторов ограниченными // Изв. вузов. Матем. 1995. № 11. С. 42-68.

[5] Арестов В. В. Приближение неограниченных операторов ограниченными и родственные экстремальные задачи // УМН. 1996. Т. 51. №6. С. 89-124.

[6] Шилов Г. Е. Математический анализ. Второй специальный курс. М.: Наука, 1965.

[7] Стейн И., Вейс Г. Введение в гармонический анализ на евклидовых пространствах. М.: Мир, 1974.

[8] Larsen R. An Introduction to the Theory of Multipliers. Berlin: Springer, 1971.

[9] Арестов В. В. Приближение операторов, инвариантных относительно сдвига // Тр. МИAH. 1975 . T. 138 . C. $43-70$.

[10] Арестов В.В. Приближение операторов типа свертки линейными ограниченньми операторами // Тр. МИАН. 1980. Т. 145. С. 3-19.

[11] Арестов В.В. Приближение инвариантных операторов // Матем. заметки. 1983. Т. 34. № 1. C. 9-29.

[12] Арестов В.В. Наилучшее приближение неограниченных операторов, инвариантных относительно сдвига, линейньми ограниченными операторами // Тр. МИАН. 1992. Т. 198. C. $3-20$.

[13] Арестов В. В. Наилучшеевосстановление операторов и родственныезадачи // Тр. МИАН. 1989. T. 189. C. $3-20$.

[14] Тайков Л.В. О наилучшем приближении в среднем некоторых классов аналитических функций // Матем. заметки. 1967. Т. 1. № 2. С. 155-162.

[15] Субботин Ю.Н. Наилучшее приближение класса функций другим классом // Матем. заметки. 1967. Т. 2. № 5. С. 495-504.

[16] Субботин Ю. Н., Тайков Л.В.Наилучшее приближение оператора дифференцирования в пространстве $L_{2} / /$ Матем. заметки. 1968. Т. 3. № 2. С. 257-264.

[17] Арестов В. В., Габушин В.Н. О приближении классов дифференцируемых функций // Матем. заметки. 1971. Т. 9. № 2. С. 105-112.

[18] Субботин Ю.Н. Связь сплайн-приближений с задачей приближения класса классом // Матем. заметки. 1971. Т. 9. № 5. С. 501-510.

[19] Никольский С. М. Приближение функций многих переменных и теоремы вложения. М.: Наука, 1977.

[20] Бесов О.В., Ильин В. П., Никольский С. М. Интегралњные представления функций и теоремы вложения. М.: Наука, 1975.

[21] Стейн И. Сингулярные интегралы и дифференциальные свойства функций. М.: Мир, 1973.

[22] Габушин В. Н. Неравенства для норм функции и ее производных в метриках $L_{p} / /$ Матем. заметки. 1967. Т. 1. №3. С. 291-298.

[23] Тимошин О.А. О наилучшем приближении дифференциальных операторов с частными производными // Матем. заметки. 1989. Т. 46. № 1. С. 78-87.

[24] Арестов В.В. Приближение линейных операторов и родственные экстремальные задачи // Тр. МИАН. 1975. Т. 138. С. 29-42.

[25] Габушин В. Н. Наилучшее приближение функционалов на некоторых множествах // Матем. заметки. 1970. Т. 8. № 5. С. 551-562.

[26] Тайков Л.В.Неравенства типа Колмогорова и наилучшие формулы численного дифференцирования // Матем. заметки. 1968. Т. 4. №2. С. 233-238.

[27] Магарил-Ильяев Г. Г., Тихомиров В. М. О неравенствах для производных колмогоровского типа // Матем. сб. 1997. Т. 188. № 12. С. 73-106.

[28] Babenko V.F., Kofanov V.A., Pichugov S. A. Exact inequalities of Kolmogorov type for multivariable functions and their applications // East J. Approx. 1997. V. 3. № 2. P. 155-186.

Уральский государственный университет им. А. М. Горького,

E-mail: Vitalii.Arestov@usu.ru 